\title{
HORI AUXILIARY SYSTEM FOR MIMAS-TETHYS
}

\author{
WAGNER SESSIN \\ Depto. de Mecânica do Vôo e Orbital \\ Instituto Tecnológico de Aeronáutica \\ 12225, São José dos Campos, SP, Brasil
}

\section{Introduction}

It is considered the problem of two natural satellites around a spheric planet. Only gravitational forces act on the system. The satellites move in a central field disturbed by the mutual atraction between them. The disturbing function is developed in power series of the small parameter (ratio of mass of satellites and planet), of the eccentricities and inclinations (assumed small) of the satellites' orbits. The mean motions are supposed to be commesurable in the ratio 2:1. It is, also, assumed that the critical angles are of inclination type. The critical angles of eccentricity type circulates and may be eliminate as fast variables like the short-periodic terms. The hamiltonian is truncated up to the second order in the inclinations.

The average hamiltonian has secular, long-periodic and resonant terms, which involve the inclinations, the critical angle and the ascending nodes of the satellites' orbits. The system of ordinary differential equations generated by this hamiltonian is called Hori Auxiliary System. Greenberg (1973) shows that with a suitable choice of the reference system the Hori Auxiliary System is solvable. Therefore, it is possible to construct a formal theory of motion for this type of resonance. This is the case of Mimas-Tethys system when the oblateness of Saturn is neglected. Here, we present the solutions of the Hori Auxiliary System for inclination type resonance using an arbitrary reference system. This study is based on the theory for the eccentricity type resonance developed by Sessin and Ferraz-Mello (1984) and same notations are used.

\section{Hori Auxiliary System}

From Sessin and Ferraz-Mello (1984) we obtain for inclination type resonance the following equations of motion

$$
\frac{d}{d t}\left(x, z_{j}\right)=\frac{\partial F}{\partial\left(\theta, \Omega_{j}\right)}, \quad \frac{d}{d t}\left(\theta, \Omega_{j}\right)=-\frac{\partial F}{\partial\left(x, z_{j}\right)} . \quad(j=1,2)
$$

The Hamiltonian is

$$
\begin{gathered}
F=\frac{1}{2} A x^{2}-\frac{1}{2} Q_{10}\left(c_{20} s_{1}^{\prime 2}+\frac{1}{2} s_{2}^{\prime 2}\right)+Q_{10} \sqrt{\frac{c_{20}}{2}} s_{1}^{\prime} s_{2}^{\prime} \cos \left(\Omega_{1}-\Omega_{2}\right) \\
+\frac{1}{2} Q_{30} c_{20} s_{1}^{\prime 2} \cos 2\left(\theta+\Omega_{1}\right)+\frac{1}{4} Q_{30} s_{2}^{\prime 2} \cos 2\left(\theta+\Omega_{2}\right) \\
-Q_{30} \sqrt{\frac{c_{20}}{2}} s_{1}^{\prime} s_{2}^{\prime} \cos \left(2 \theta+\Omega_{1}+\Omega_{2}\right)
\end{gathered}
$$


where $A=\frac{4 F_{20}}{\left|x_{20}\right|}, Q_{10}, Q_{30}$ and $c_{20}=\left|c_{2}\right|$ are physical constants(time scale has been changed by the factor $\left.\left|2 x_{20}\right|\right)$.

Introducing the non-canonical variables

$$
\begin{aligned}
& P_{1}=\frac{1}{2} p_{1}+\sqrt{\frac{c_{20}}{2}} p_{2}, \quad Q_{1}=\frac{1}{2} q_{1}+\sqrt{\frac{c_{20}}{2}} q_{2}, \\
& P_{2}=\sqrt{\frac{c_{20}}{2}} p_{1}-\frac{1}{2} p_{2}, \quad Q_{2}=\sqrt{\frac{c_{20}}{2}} q_{1}-\frac{1}{2} q_{2}
\end{aligned}
$$

with

$$
\begin{gathered}
p_{j}=\sqrt{-2 \rho_{j}} \sin \sigma_{j}, \quad q_{j}=\sqrt{-2 \rho_{j}} \cos \sigma_{j}, \quad s_{j}^{\prime}=\sqrt{-2 \rho_{j}}, \\
x=\rho_{0}-\frac{1}{2} \sum_{j=1}^{2}\left(p_{j}^{2}+q_{j}^{2}\right), \quad \rho_{j}=-\frac{1}{2}\left(p_{j}^{2}+q_{j}^{2}\right), \\
\sigma_{j}=\theta+\Omega_{j}, \quad \theta_{j}=\lambda_{1}-2 \lambda_{2}, \quad z_{j}=\rho_{j}, \\
x=\rho_{0}+\rho_{1}+\rho_{2},
\end{gathered}
$$

where $\rho_{0}$ is a first integral, the new equations of motion are

$$
\begin{gathered}
\frac{d}{d t} P_{1}=-F_{02} x Q_{1}, \quad \frac{d}{d t} Q_{1}=F_{02} x P_{1}, \\
\frac{d}{d t} P_{2}=-F_{02} x Q_{2}+\left(c_{20}+\frac{1}{2}\right)\left(Q_{30}-Q_{10}\right) Q_{2} \\
\frac{d}{d t} Q_{2}=F_{02} x P_{2}+\left(c_{20}+\frac{1}{2}\right)\left(Q_{30}+Q_{10}\right) P_{2} \\
\frac{d}{d t} \theta=-F_{02} x, \quad \frac{d}{d t} x=-4 Q_{30} P_{2} Q_{2} .
\end{gathered}
$$

There are four constants of integration $\left(E, G, s_{10}, \Omega_{10}\right)$ defined by the equations

$$
\begin{gathered}
\frac{1}{2} \frac{A}{Q_{30}} x^{2}+\frac{Q_{10}\left(c_{20}+\frac{1}{2}\right)}{Q_{30}} x+Q_{2}^{2}-P_{2}^{2}=E, \quad P_{2}^{2}+Q_{2}^{2}=-\left(c_{20}+\frac{1}{2}\right)(x+G) \\
P_{1}=s_{10} \sin \left(\theta+\Omega_{10}\right), \quad Q_{1}=s_{10} \cos \left(\theta+\Omega_{10}\right)
\end{gathered}
$$

and the system is solvable. The solutions are functions of $x(t)$ which is the solution of the differential equation

$$
\frac{d}{d t} x= \pm \sqrt{-P(x)}
$$

where

$$
\begin{gathered}
P(x)=A^{2} x^{4}+\left\{4 A\left(c_{20}+\frac{1}{2}\right) Q_{10}\right\} x^{3} \\
+\left\{4\left(c_{20}+\frac{1}{2}\right)^{2}\left(Q_{10}^{2}-Q_{30}^{2}\right)-A Q_{30} E\right\} x^{2}
\end{gathered}
$$




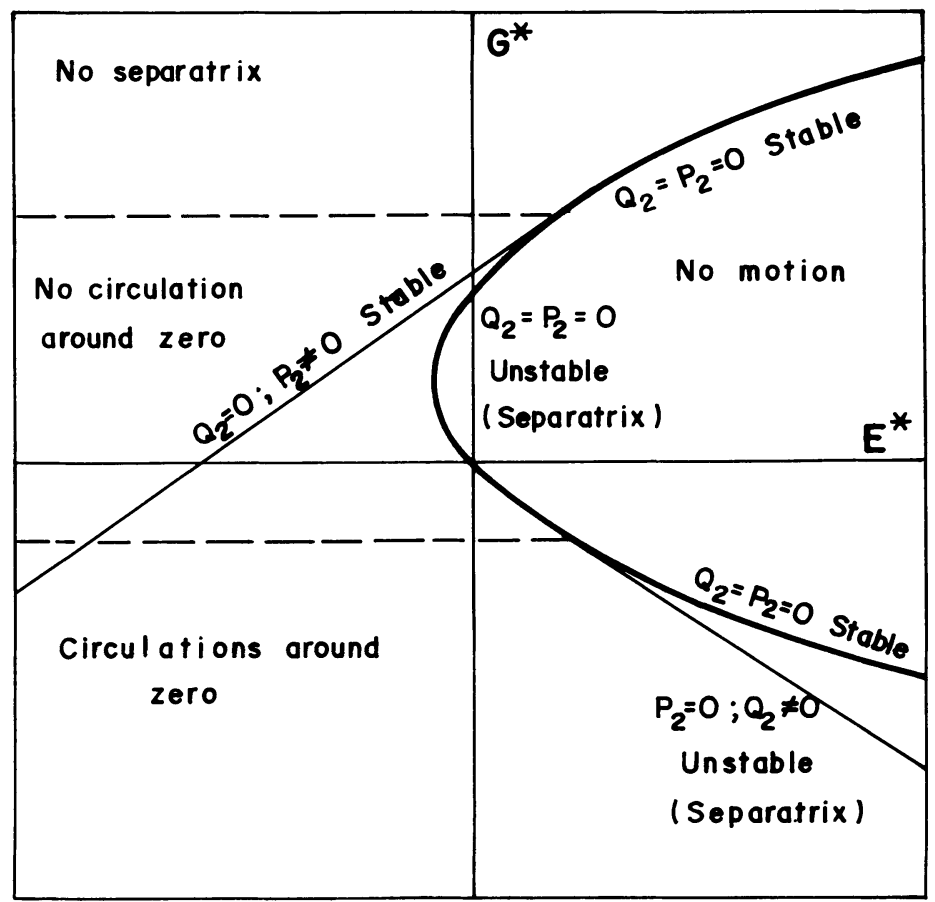

Fig. 1. Regions of the plane $\left(E^{*}, G^{*}\right)$

$$
\begin{gathered}
-8\left(c_{20}+\frac{1}{2}\right) Q_{10} Q_{30}\left\{\left(c_{20}+\frac{1}{2}\right) G+E\right\} x \\
-4 Q_{30}^{2}\left\{\left(c_{20}+\frac{1}{2}\right)^{2} G^{2}-E^{2}\right\} .
\end{gathered}
$$

Therefore, the Hori auxiliary system with inclination type resonance is completely integrable. The solutions in the plane $\left(Q_{2}, P_{2}\right)$ are similar to those for the eccentricity type resonance of the second order (Jefferys, 1966; Lemaitre, 1984). Similarly to what was done by Sessin and Ferraz-Mello(1984) the families of periodic orbits in the plane $\left(E^{*}, G^{*}\right)$ may be obtained, where

$$
E^{*}=\frac{E}{\left(c_{20}+\frac{1}{2}\right) Q_{10}^{2}}, G^{*}=\frac{G}{\left(c_{20}+\frac{1}{2}\right) Q_{10}} .
$$

The roots of the polynomial $P(x)$ may be calculated and the solutions of the auxiliary system determined. The plane $\left(E^{*}, G^{*}\right)$ is presented in Figure 1. In Figure 2 the fixed points are the trivial periodic solutions belonging to the families represented in Figure 1. As it is well known, the types of motion that appear in Figure 2 are: libration, circulation (external and internal) and asymptotic motion (separatrix). 

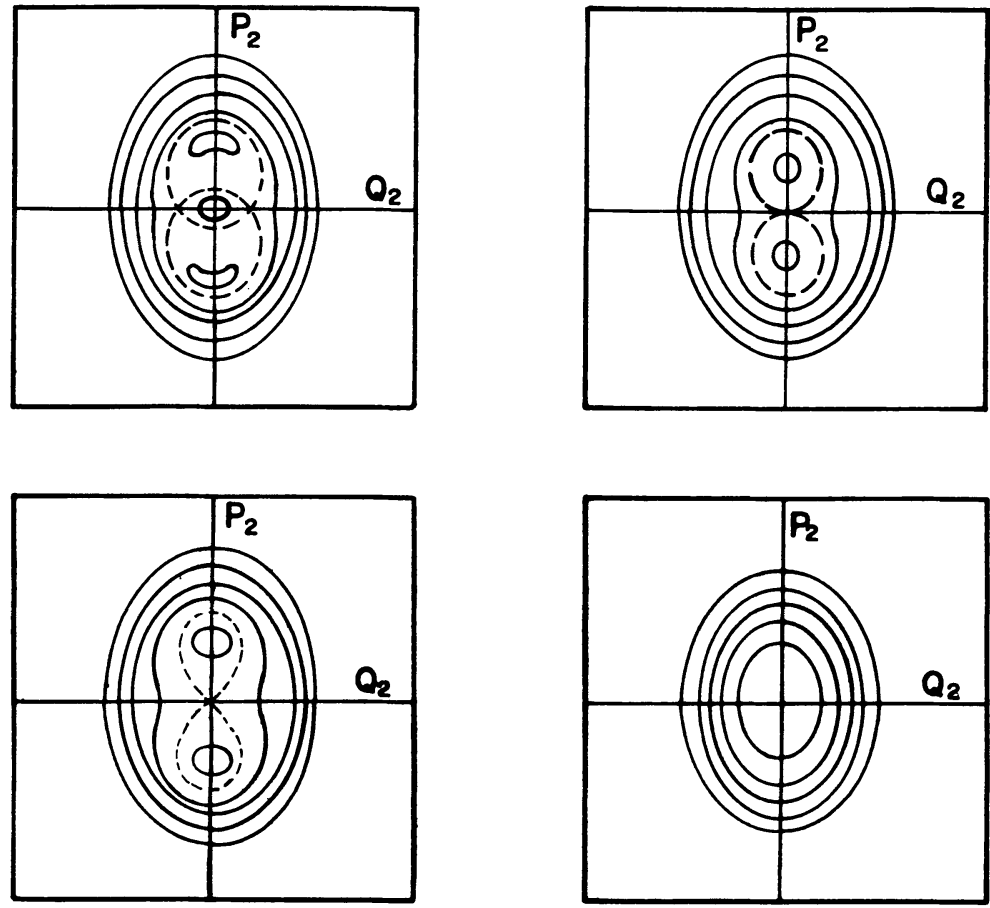

Fig. 2. Types of Motion in the Plane $\left(Q_{2}, P_{2}\right)$

The different types of motion are separated by the separatrices (it must be noted that for $x=E=G=0$ the unstable periodic solution becomes an equilibrium point). Finally, these solutions of the Hori auxiliary system for inclination type resonance give the first approximation for the construction of formal theory for this type of problem.

\section{Acknowledgements}

The author thanks to FAPESP by the financial aid.

\section{References}

Greenberg, R.:1973, Mon. Not. R. astr. Soc. 165, 305.

Jefferys, W.H.:1966, Astron. J. 71, 306.

Lemaitre, A.:1984, Celest. Mech. 32, 109.

Sessin, W. and Ferraz-Mello, S.:1984, Celest. Mech. 32, 307. 